\title{
Metabotropic Glutamate Subtype 5 Receptors Are Quantified in the Human Brain with a Novel Radioligand for PET
}

Amira K. Brown, Yasuyuki Kimura, Sami S. Zoghbi, Fabrice G. Siméon, Jeih-San Liow, William C. Kreisl, Andrew Taku, Masahiro Fujita, Victor W. Pike, and Robert B. Innis

Molecular Imaging Branch, National Institute of Mental Health, Bethesda, Maryland

We developed a radioligand, 3-fluoro-5-(2-(2-18 F-(fluoromethyl)thiazol-4-yl)ethynyl)benzonitrile ( $\left.{ }^{18} \mathrm{~F}-\mathrm{SP} 203\right)$, for metabotropic glutamate subtype 5 (mGluR5) receptors that showed both promising (high specific binding) and problematic (defluorination) imaging characteristics in animals. The purposes of this initial evaluation in human subjects were to determine whether ${ }^{18} \mathrm{~F}-\mathrm{SP} 203$ is defluorinated in vivo (as measured by uptake of radioactivity in the skull) and to determine whether the uptake in the brain can be quantified as distribution volume relative to concentrations of ${ }^{18} \mathrm{~F}-\mathrm{SP} 203$ in plasma. Methods: Seven healthy subjects were injected with ${ }^{18} \mathrm{~F}-\mathrm{SP} 203(323 \pm 87 \mathrm{MBq})$ and scanned over $5 \mathrm{~h}$, with rest periods outside the camera. The concentrations of ${ }^{18} \mathrm{~F}-\mathrm{SP} 203$, separated from radiometabolites, were measured in arterial plasma. Results: The skull was difficult to visualize on PET images in the initial $2 \mathrm{~h}$, because of high radioactivity in the brain. Although radioactivity in the skull and adjacent cortex showed some cross-contamination, the concentration of radioactivity in the skull was less than half of that in the adjacent cortex during the initial $2 \mathrm{~h}$. Modeling of regional brain and plasma data showed that a 2-tissue-compartment model was superior to a 1-tissuecompartment model, consistent with measurable amounts of both receptor-specific and nonspecific binding. The concentrations of activity in the brain measured with PET were consistently greater than the modeled values at late but not early time points and may well have been caused by the slow accumulation of radiometabolites in the brain. To determine an adequate time for more accurate measurement of distribution volume, we selected a scan duration (i.e., 2 h) associated with maximal or near-maximal identifiability. Distribution volume was well identified ( $\sim 2 \%$ ) by only $2 \mathrm{~h}$ (and even just 1 ) of image acquisition. Conclusion: This initial evaluation of ${ }^{18} \mathrm{~F}-\mathrm{SP} 203$ in healthy human subjects showed that defluorination is relatively small and that brain uptake can be robustly calculated as distribution volume. The values of distribution volume were well identified and had relatively small variation in this group of 7 subjects. These results suggest that ${ }^{18} \mathrm{~F}-\mathrm{SP} 203$ will have good sensitivity to measure mGluR5 receptors for both within-subject studies (e.g., receptor occupancy) and between-subject studies (e.g., patients vs. healthy subjects).

Received Jul. 24, 2008; revision accepted Aug. 18, 2008.

For correspondence or reprints contact: Robert B. Innis, Molecular Imaging Branch, National Institute of Mental Health, 31 Center Dr., Room B2/B37 Bethesda, MD 20892-2035.

E-mail: robert.innis@nih.gov

COPYRIGHT ( 2008 by the Society of Nuclear Medicine, Inc.
Key Words: molecular imaging; glutamate neurotransmission; PET

J Nucl Med 2008; 49:2042-2048

DOI: 10.2967/jnumed.108.056291

$\mathbf{M}$ etabotropic glutamate receptors (mGluRs) are coupled to $G$ proteins and modulate intracellular second messenger systems. Binding of agonists to the mGluR5 receptor subtype stimulates phospholipase $\mathrm{C}$, which results in phosphoinositide hydrolysis and mobilization of intracellular calcium (1). Studies using animal models suggest mGluR5 function may be impaired in several human disorders, including anxiety, schizophrenia, substance abuse, and fragile $\mathrm{X}$ syndrome (2). In response to these animal studies, several drugs that modulate mGluR5 function are being developed. The drug target on mGluR5 is distinct from the glutamate binding site. Instead, the drug target is an allosteric (or noncompetitive) site that can either increase or decrease function via positive or negative allosteric modulation, respectively (3).

Hamill et al. (4) have reported 3 promising PET radioligands for the allosteric site on the mGluR5 receptor, 1 labeled with ${ }^{11} \mathrm{C}$ and 2 labeled with ${ }^{18} \mathrm{~F}$. Each radioligand is a diaryl alkyne with high affinity and selectivity for mGluR5. The 3 radioligands have good uptake in monkey brains $(200 \%-400 \%$ standarized uptake value [SUV]), which may be blocked by an mGluR5-selective nonradioactive ligand, MTEP. These radioligands have not yet been studied in human subjects. Ametamey et al. (5), however, developed another radioligand that has been studied in humans. This radioligand, ${ }^{11} \mathrm{C}$-ABP688, is also a diaryl alkyne and has promising kinetic characteristics to quantify receptors in the brain. That is, the peak uptake is achieved early and is moderately high (150\%-200\% SUV), with rapid washout (6).

We recently developed a radioligand for the mGluR5 receptor, 3-fluoro-5-(2-(2- ${ }^{18}$ F-(fluoromethyl)thiazol-4-yl) ethynyl)benzonitrile, designated ${ }^{18} \mathrm{~F}-\mathrm{SP} 203$ or compound (3) in the study by Siméon et al. (7). ${ }^{18}$ F-SP203 is a negative allosteric modulator and has unusually high affinity (40 pM) 
for the mGluR5 receptor and high potency $(0.7 \mathrm{pM})$ in a phosphoinositol hydrolysis assay.

${ }^{18}$ F-SP203 has both promising and problematic characteristics in animal studies (7). Promising characteristics include high uptake into monkey brains, moderate rate of washout, and displacement by an mGluR5-selective nonradioactive ligand. The problematic characteristics derive from metabolism via defluorination. Under in vitro conditions, ${ }^{18} \mathrm{~F}-\mathrm{SP} 203$ is defluorinated in rat brains and in monkey blood. This defluorination is evident in vivo from the trapping of the ${ }^{18} \mathrm{~F}$-fluoride ion in rat brains and in monkey skulls. Although ${ }^{18}$ F-SP203 had negligible defluorination during in vitro incubation with human brain and blood, we did not know whether other human organs, such as the liver, would defluorinate the radioligand in vivo.

The present study reports the initial evaluation of ${ }^{18} \mathrm{~F}$-SP203 in human subjects. We sought to determine whether ${ }^{18} \mathrm{~F}$-SP203 is defluorinated in vivo (as measured by uptake of radioactivity in the skull) and whether ${ }^{18} \mathrm{~F}-\mathrm{SP} 203$ uptake in the brain can be quantified as distribution volume relative to serial concentrations of ${ }^{18} \mathrm{~F}-\mathrm{SP} 203$ in plasma.

\section{MATERIALS AND METHODS}

\section{Radioligand Preparation}

${ }^{18} \mathrm{~F}-\mathrm{SP} 203$ was prepared from the bromomethyl analog of SP203 and the ${ }^{18} \mathrm{~F}$-fluoride ion, as previously described (7). The radioligand was prepared according to our Investigational New Drug Application 78,260, submitted to the U.S. Food and Drug Administration (a copy of which is available at http://pdsp.med. unc.edu/snidd/).

${ }^{18} \mathrm{~F}-\mathrm{SP} 203$ had radiochemical purity greater than $98 \%$ and specific activity at the time of injection of $156 \pm 54 \mathrm{GBq} / \mu \mathrm{mol}$.

\section{Human Subjects}

Seven subjects ( 2 women and 5 men; age, $37 \pm 11 \mathrm{y}$; body weight, $83 \pm 9 \mathrm{~kg}$ ) participated in the study. All subjects were free of current medical and psychiatric illness on the basis of history, physical examination, electrocardiogram, urinalysis including drug screening, and blood tests (complete blood count, serum chemistries, thyroid function test, and antibody screening for syphilis, HIV, and hepatitis B). Repeated urinalysis and blood tests were acquired 7-24 $\mathrm{h}$ after injection of the radioligand.

\section{PET Scans}

After injection of ${ }^{18} \mathrm{~F}$-SP203 $(323 \pm 87 \mathrm{MBq})$, 3-dimensional dynamic PET images were acquired on an Advance camera (GE Healthcare) for $300 \mathrm{~min}$ in 45 frames of increasing duration from $30 \mathrm{~s}$ to $5 \mathrm{~min}$. All PET images were reconstructed with a resolution of approximately $7.5 \mathrm{~mm}$ and corrected for attenuation and scatter. Subjects had 3 rest periods ( $30 \mathrm{~min}$ each) outside the camera, beginning at approximately 120, 180, and $240 \mathrm{~min}$ after injection of the radioligand. Head movement was corrected after the scan by realigning all images from each subject using Statistical Parametric Mapping (version 5; Wellcome Department of Cognitive Neurology).

\section{MRI}

To identify brain regions, MR images of $1.2-\mathrm{mm}$ contiguous slices were obtained with a 1.5-T Signa device (GE Healthcare).
Three sets of axial images were obtained using spoiled gradient recall acquisition in the steady state (repetition time, $12.4 \mathrm{~ms}$; echo time, $5.3 \mathrm{~ms}$; flip angle, $20^{\circ}$; and matrix, $256 \times 256$ ). Two of the $7 \mathrm{MR}$ images were obtained using a 3.0-T Achieva device (Philips) with the following parameters: scans of 1-mm contiguous slices were obtained, and 3 sets of axial images were acquired with a repetition time of $20 \mathrm{~ms}$, echo time of $4.9 \mathrm{~ms}$, and flip angle of $30^{\circ}$ ). These 3 MRI sets from both the GE and Philips devices were averaged and then coregistered to the PET images.

\section{Measurement of ${ }^{18} \mathrm{~F}-\mathrm{SP} 203$ in Plasma}

Blood samples $(1.5 \mathrm{~mL}$ each) were drawn from the radial artery at 15 -s intervals until $120 \mathrm{~s}$, followed by $1.5-\mathrm{mL}$ samples each at 3,5 , 10, 20, 30, and $45 \mathrm{~min} ; 3-\mathrm{mL}$ samples each were then drawn at 60 , $90,120,150$, and $180 \mathrm{~min}$. To determine the stability of ${ }^{18} \mathrm{~F}-\mathrm{SP} 203$ in vitro, approximately $15 \mathrm{kBq}$ of radioligand were incubated with blood and plasma from each subject for $30 \mathrm{~min}$ at room temperature. Radioactivity in plasma and the in vitro standards was extracted into acetonitrile and analyzed by reverse-phase chromatography with a Novapak-C18 column $(100 \times 8 \mathrm{~mm}$; Waters Corp. $)$, using a radial compression module (RCM-100; Waters) with a sentry precolumn and a mobile phase of methanol:water:triethylamine (70:30:0.1 by volume) at a flow rate of $2.0 \mathrm{~mL} / \mathrm{min}$. Plasma-free fraction was measured by ultrafiltration as previously described (8).

\section{Image Analysis}

Preset volumes of interest were positioned on the subject's MR image and then transferred to the coregistered PET images by the following methods. Frames $4-45$ of the PET scan were averaged to create a single image showing good delineation of cerebral cortices. The average MR image from 3 acquisitions of each subject was coregistered to the average PET image using Statistical Parametric Mapping (version 5). Both MR and all PET images were then spatially normalized to a standard anatomic orientation (Montreal Neurologic Institute space) on the basis of transformation parameters from the MR images. Preset volumes of interest (9) were applied to the spatially normalized PET images to extract time-activity curves for the following 10 regions: frontal $\left(432 \mathrm{~cm}^{3}\right)$, parietal $\left(247 \mathrm{~cm}^{3}\right)$, occipital $\left(172.3 \mathrm{~cm}^{3}\right)$, temporal $\left(251 \mathrm{~cm}^{3}\right)$, and medial temporal $\left(36 \mathrm{~cm}^{3}\right)$ cortices; caudate $\left(16 \mathrm{~cm}^{3}\right)$; putamen $\left(17 \mathrm{~cm}^{3}\right)$; cingulate $\left(28 \mathrm{~cm}^{3}\right)$; thalamus $\left(17 \mathrm{~cm}^{3}\right)$; and cerebellum $\left(195 \mathrm{~cm}^{3}\right)$. For the skull, 2 regions were drawn bilaterally over the external table of the parietal bone on the coregistered MR image of each subject and applied to the PET images. The total volume of the 2 regions was approximately $0.85 \mathrm{~cm}^{3}$, and the distance from the surface of the cortex was approximately $5 \mathrm{~mm}$.

\section{Calculation of Distribution Volume Using Radiometabolite-Corrected Input Function}

Distribution volume is an index of receptor density and is equal to the ratio at equilibrium of the concentration of radioligand in tissue to that in plasma. Total distribution volume $\left(V_{\mathrm{T}}\right)$ includes the concentrations of all radioligand in tissue, which is composed of specific binding (i.e., receptor-bound) and nondisplaceable uptake (i.e., nonspecifically bound and free radioligand in tissue water). The equilibrium value of $V_{\mathrm{T}}$ was calculated from measurements over time of radioactivity in the brain and of the concentrations of radioligand in arterial plasma. The input function was analyzed as a linear interpolation of the concentrations of ${ }^{18} \mathrm{~F}-\mathrm{SP} 203$ before the peak and a triexponential fit of concentrations after the peak. Rate constants $\left(K_{1}, k_{2}, k_{3}\right.$, and $\left.k_{4}\right)$ in standard 1 - and 
2-tissue-compartment models (10) were calculated with weighted least squares and the Marquardt optimizer. Brain data of each frame were weighted by assuming that the SD of the data was proportional to the inverse square root of noise equivalent counts. To correct the brain data for its vascular component, radioactivity in serial whole blood was measured and then subtracted from the PET measurements, assuming that blood constitutes $5 \%$ of total brain volume. Image and kinetic analyses were performed using pixelwise modeling software (PMOD 2.9; PMOD Technologies Ltd.) (11).

\section{Statistical Analysis}

Goodness of fit by nonlinear least-squares analysis was evaluated using the Akaike information criterion (AIC) (12) and the model selection criterion (MSC). The most appropriate model is the one with the smallest AIC and the largest MSC values. Compartment models were compared with $\mathrm{F}$ statistics that accounted for the number of variables in each model (13). A value of $P$ less than 0.05 was considered significant for $F$ statistics.

The identifiability of the kinetic variables was calculated by the compartmental fitting as the SE, which itself reflects the diagonal of the covariance matrix (14). Identifiability is expressed as a percentage and equals the ratio of the SE of the rate constant divided by the value of the rate constant itself. A lower percentage indicates better identifiability, because a smaller SE would be a smaller percentage of the rate constant.

Group data are expressed as mean $\pm \mathrm{SD}$.

\section{RESULTS}

\section{Pharmacologic Effects}

On the basis of subject reports, electrocardiogram, blood pressure, pulse, and respiration rate, ${ }^{18} \mathrm{~F}-\mathrm{SP} 203$ was determined to have caused no pharmacologic effects during the 5-h scans. In addition, no effects were noted in any of the blood and urine tests acquired 7-24 $\mathrm{h}$ after radioligand injection. The injected activity of ${ }^{18} \mathrm{~F}-\mathrm{SP} 203$ was $323 \pm 87$ $\mathrm{MBq}$, which corresponded to $2.3 \pm 0.8 \mathrm{nmol}$ of SP203 ( $n=7$ injections in 7 subjects).

\section{Radioactivity in Brain and Skull}

After injection of ${ }^{18} \mathrm{~F}-\mathrm{SP} 203$, all subjects showed high concentrations of radioactivity in the brain that decreased gradually. Radioactivity in the brain peaked at 5 min and was approximately $580 \% \mathrm{SUV}$ in most regions, including the temporal cortex and thalamus (Figs. 1 and 2). Brain activity decreased to $50 \%$ of peak at between 90 and $180 \mathrm{~min}$ in the 7 subjects.

Because of the high concentrations of radioactivity in the brain, we could not easily identify the skull on the PET images, especially those acquired in the initial $120 \mathrm{~min}$. For this reason, we quantified uptake in the skull by identifying this structure on MRI and sampling data from the coregistered PET images. In fact, the skull did have uptake of radioactivity, reaching $100 \%$ SUV within a few minutes of injection and gradually increasing to $150 \% \mathrm{SUV}$ at $5 \mathrm{~h}$. Because of the proximity to the brain, some of the skull uptake was actually spillover from the brain. However, not all the radioactivity was spillover, because activity in the

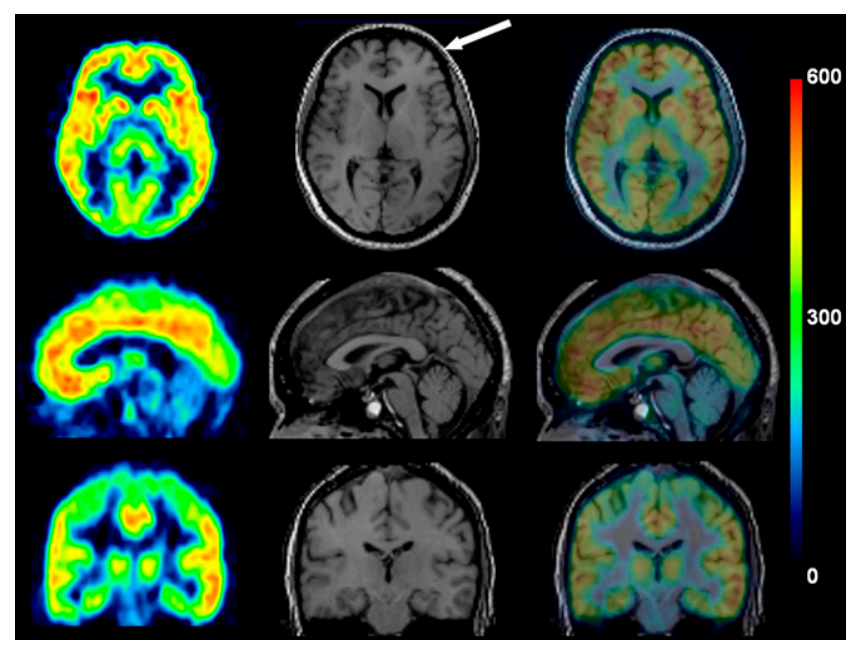

FIGURE 1. PET and MR images from healthy subject. PET images from 60 to 180 min after injection of ${ }^{18} \mathrm{~F}-\mathrm{SP} 203$ were summed; pixel values represent mean concentration of radioactivity (\%SUV). Coregistered MR images are in middle column, and fused PET and MR images are in right column. Arrow points to subcutaneous fat, which has high signal intensity on this T1weighted image. Skull lies in dark area between this highintensity signal and brain.

skull slowly increased after $10 \mathrm{~min}$, whereas that in the brain decreased (Fig. 2).

\section{Plasma Clearance}

The concentration of ${ }^{18} \mathrm{~F}-\mathrm{SP} 203$ peaked and then rapidly declined, following a curve that was well fitted as a triexponential function, showing average half-lives of $0.5,5$, and 59 min (Fig. 3A). Calculated as the partial area under the concentration-versus-time curve, these 3 half-lives accounted for $27 \%, 32 \%$, and $40 \%$, respectively, of the area under the curve from peak to infinity.

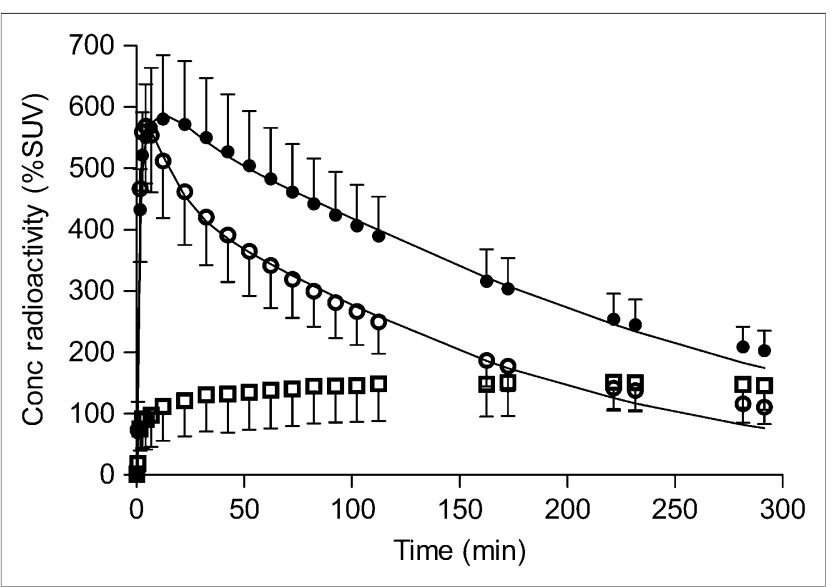

FIGURE 2. Average time course of radioactivity in brain after injection of ${ }^{18} \mathrm{~F}-\mathrm{SP} 203$. Concentrations of radioactivity are shown for brain regions with highest (temporal cortex, 0 ) and lowest (thalamus, $\bigcirc$ ) concentrations of radioactivity and skull $(\square)$. Measured brain data were fit with 2-tissue-compartment model without constraint (solid line). Data represent mean \pm SD of all 7 subjects. Conc $=$ concentration. 

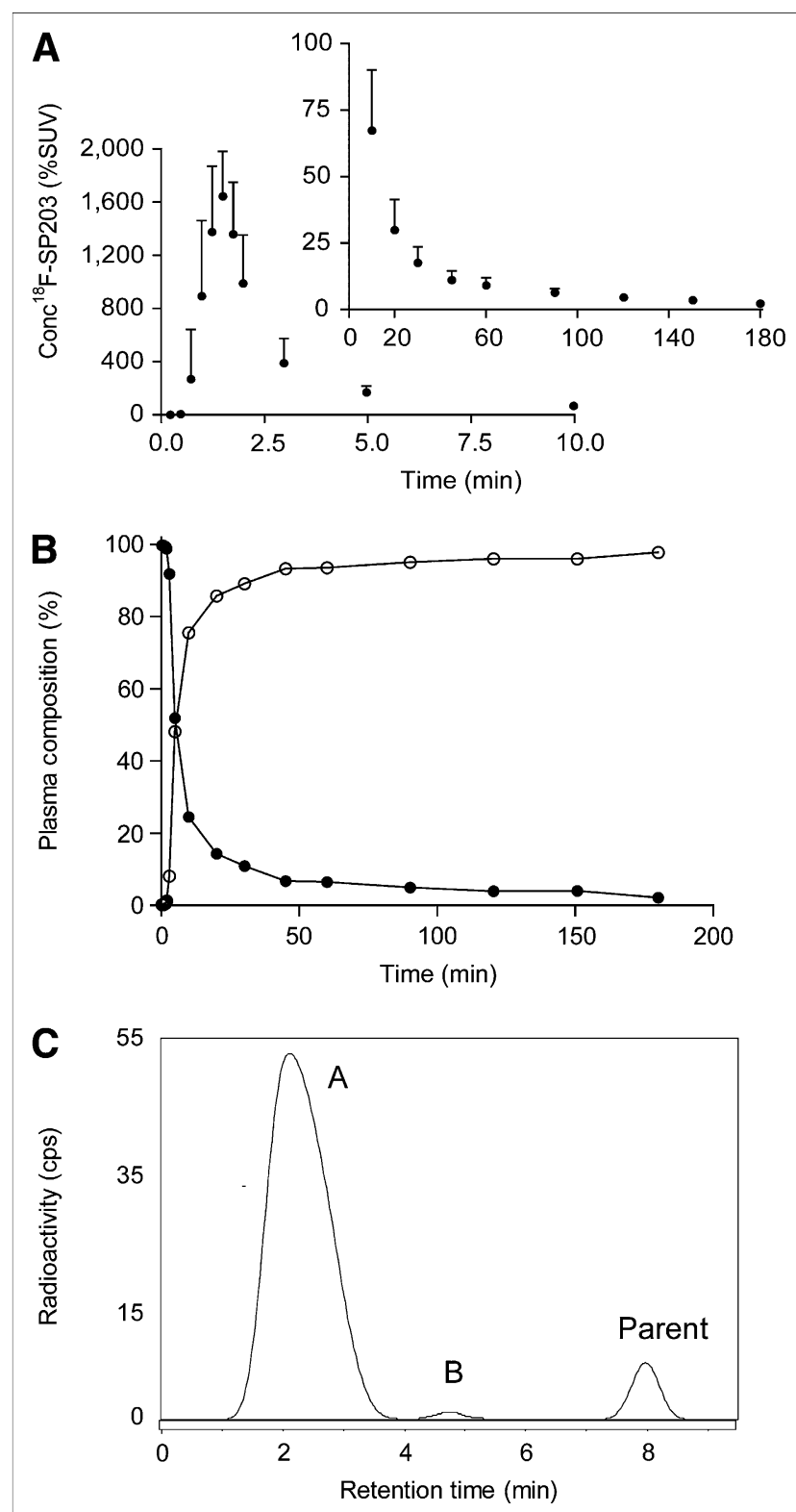

FIGURE 3. Concentration of ${ }^{18} \mathrm{~F}-\mathrm{SP} 203$ and its percentage composition in arterial plasma. (A) Average concentration (\%SUV) of ${ }^{18} \mathrm{~F}-\mathrm{SP} 203$ in arterial plasma is plotted vs. time after injection. Value for $10 \mathrm{~min}$ is shown on both graphs, which differ in range of $y$-axis. (B) Percentage composition of plasma that represents parent radioligand $(O)$ and radiometabolites $(O)$ are plotted vs. time after injection. (C) This radiochromatogram illustrates plasma composition at $45 \mathrm{~min}$ after injection of ${ }^{18} \mathrm{~F}-\mathrm{SP} 203$. One major radiometabolite $(\mathrm{A} ; 92 \%)$ eluted in void volume of column followed by minor one $(\mathrm{B} ; 1 \%)$ and parent ${ }^{18} \mathrm{~F}-\mathrm{SP} 203$ (7\%). Radiometabolites A and B are both more polar than parent. Conc $=$ concentration.

One major radiometabolite of ${ }^{18} \mathrm{~F}$-SP203 appeared quickly in plasma (Figs. 3B and 3C). The radiometabolite was much less lipophilic than was the parent and eluted near the void volume on reverse-phase high-performance liquid chromatography. Although not further identified, the radiometabolite could have been the ${ }^{18} \mathrm{~F}$-fluoride ion.
The in vitro stability of ${ }^{18} \mathrm{~F}-\mathrm{SP} 203$ was measured by incubating the radioligand with blood and plasma for $30 \mathrm{~min}$ at room temperature. The stability was $93 \% \pm$ $2.5 \%(n=7)$ in whole blood and $94 \% \pm 2.6 \%(n=7)$ in plasma. This stability is similar to what we typically measure for several other radioligands $(>90 \%)$. Furthermore, although these in vitro standards were incubated for $30 \mathrm{~min}$ at room temperature, the plasma samples acquired from subjects after injection of the radioligand are processed within only 5-10 min. Thus, the radioligand had negligible metabolism in vitro after blood samples were withdrawn from the artery.

The plasma-free fraction of ${ }^{18} \mathrm{~F}-\mathrm{SP} 203$ was $5.2 \% \pm 0.2 \%$ in the 7 subjects.

\section{Kinetic Analysis}

For all subjects, the unconstrained 2-tissue-compartment model provided a significantly better fit than did the 1-tissuecompartment model, consistent with the presence of significant amounts of both specific and nonspecific binding in the human brain. Although the 1-tissue model estimated $K_{1}, k_{2}$, and $V_{\mathrm{T}}$ with reasonable identifiability $(3 \%-5 \%)$, the curves significantly deviated from the measured brain data and had high AIC scores (330-375) and low MSC scores (1-2). The 2-tissue-compartment model fitted the data better than did the 1-tissue-compartment model and had low AIC scores (218-258) and high MSC scores (4-5). Finally, the F test showed that the 2-tissue-compartment model gave a statistically better fit than did the 1-tissue compartment model in all regions for all subjects.

For the 2-tissue-compartment model, we assessed the utility of constraining the value of nondisplaceable uptake $\left(V_{\mathrm{ND}}=K_{1} / k_{2}\right)$ in all regions to a single value determined from a composite of all 10 brain regions. This constraint improved the identifiability of $V_{\mathrm{T}}$ slightly in some regions. However, in several regions, the F test showed that the unconstrained model gave a statistically better fit and low AIC scores and high MSC scores. For this reason, we used the unconstrained 2-tissue-compartment model for additional analyses.

The unconstrained 2-tissue-compartment model consistently deviated at later time points from the measured values in all brain regions and in all subjects. That is, the measured values after $200 \mathrm{~min}$ were always higher than those projected from modeling (Fig. 2). This deviation was paralleled by the increasing value of $V_{\mathrm{T}}$ for scan durations greater than $200 \mathrm{~min}$ (Fig. 4A). These results are consistent with the accumulation of radiometabolites in the brain, especially at later time points. That is, the measured brain activity was greater than that predicted from using only the parent radioligand in plasma as the input function. Because the long scan duration of 300 min might have provided inaccurate results, we chose a shorter duration that would still provide good identifiability of $V_{\mathrm{T}}$. Identifiability reached a minimal or near-minimal value of approximately $1 \%$ with 120 min of data (Fig. 4A). We then assumed that the 120-min data provided the optimal 

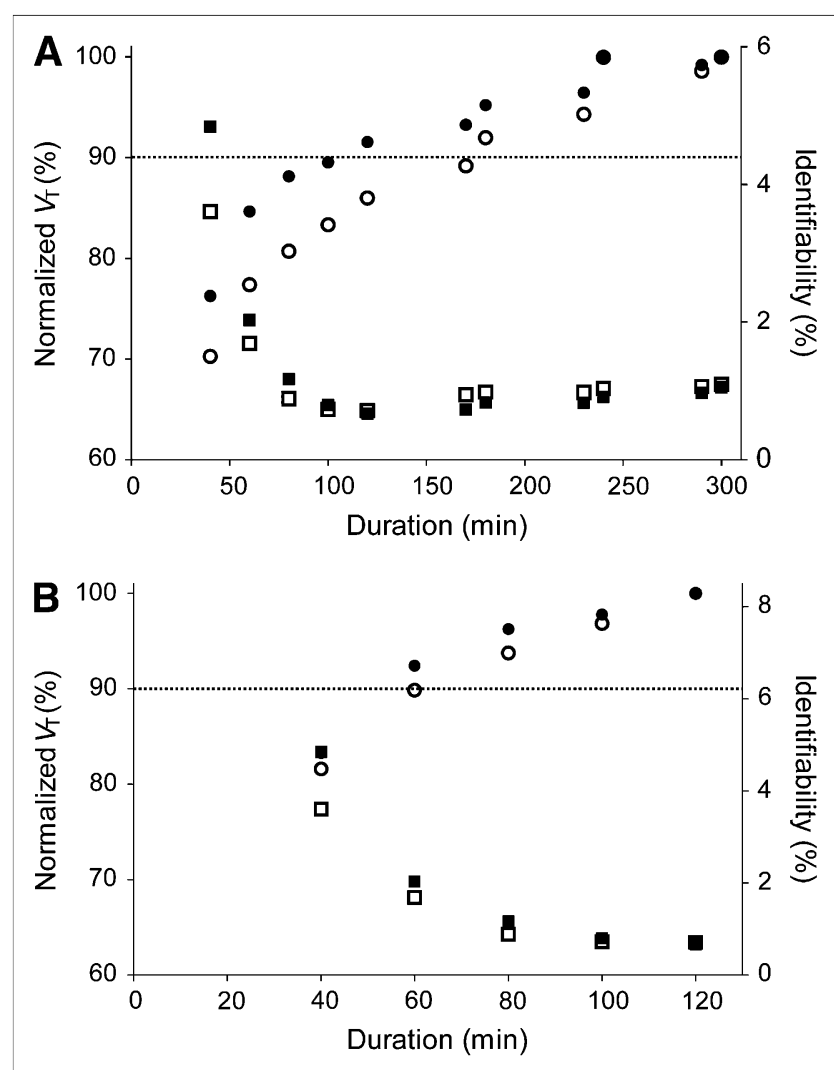

FIGURE 4. Value of $\mathrm{V}_{\mathrm{T}}$ and identifiability as function of duration of image acquisition using either $300(\mathrm{~A})$ or 120 (B) min as terminal points. $V_{\mathrm{T}}$ was calculated for temporal cortex $(\mathbf{O})$ and thalamus $(\bigcirc)$ using unconstrained 2-tissue-compartment model. Scans were analyzed using brain data from time 0 to specified time on $x$-axis. $V_{\mathrm{T}}$ was expressed as percentage of terminal value. That is, terminal value of $V_{T}$ calculated from either entire $300 \mathrm{~min}$ of scanning (A) or only initial $120 \mathrm{~min}(\mathrm{~B})$. Values of normalized $V_{\mathrm{T}}$ are shown with circles for temporal cortex $(\Theta)$ and thalamus $(\bigcirc)$ and use $y$-axis on left. Corresponding values of identifiability are shown with squares $(\square, \square)$ and use $y$-axis on right.

analysis and explored whether even shorter scan durations would be adequate. If $120 \mathrm{~min}$ of data provided the correct value, then only 60 min of data estimated $V_{\mathrm{T}}$ within $10 \%$ of this correct value and with excellent identifiability of $2 \%$ (Fig. 4B).

From these analyses, we determined that the optimal method of quantitation should use the unconstrained, 2tissue-compartment model of the initial $120 \mathrm{~min}$ of data, and we calculated $V_{\mathrm{T}}$ in 10 regions in all 7 subjects using this method. The curves had excellent fit, with high values of AIC $(\sim 110)$ and low values of MSC $(\sim 5)$ (Table 1$)$. The rate constants $\left(K_{1}, k_{2}, k_{3}\right.$, and $\left.k_{4}\right)$ had good identifiability $(<11 \%)$ for individual variables, and $V_{\mathrm{T}}$ had excellent identifiability $(<2 \%)$ for a composite ratio of variables. The regional values of $V_{\mathrm{T}}\left(\mathrm{mL} \times \mathrm{cm}^{-3}\right)$ were highest in the neocortex $(\sim 20-26)$ and lowest in the thalamus $(\sim 15)$ and cerebellum $(\sim 14)$, consistent with the known distribution of mGluRs in the human brain (15). Furthermore, the variability of $V_{\mathrm{T}}$ values was low in this sample of 7 healthy subjects. For example, the coefficient of variation ( $=\mathrm{SD} /$ mean) of $V_{\mathrm{T}}$ in most brain regions was between $10 \%$ and $15 \%$ (Table 1). Finally, the estimated ratio of specific to nondisplaceable uptake $\left(B P_{\mathrm{ND}}\right)$ was relatively high $(\sim 5)$ in most brain regions, but this value may not be accurate. That is, the identifiability of $B P_{\mathrm{ND}}$ was approximately $10 \%$ in most regions, which is relatively poor for this composite variable $\left(=k_{3} / k_{4}\right)$.

\section{DISCUSSION}

This initial evaluation of ${ }^{18} \mathrm{~F}-\mathrm{SP} 203$ in healthy human subjects showed that brain uptake can be robustly calculated as distribution volume, despite a modest amount of defluorination and uptake of radioactivity in the skull. Brain uptake was high (peak uptake, $\sim 580 \%$ ) and washed out moderately rapidly, despite the high in vitro affinity of SP203. Radiometabolites may have slowly accumulated in the brain, thereby causing long image acquisitions $(5 \mathrm{~h})$ to overestimate $V_{\mathrm{T}}$. Imaging for between 1 and $2 \mathrm{~h}$ was adequate to calculate $V_{\mathrm{T}}$ with excellent identifiability $(\sim 2 \%)$.

\section{Adequate Scan Length}

SP203 has unusually high affinity for mGluR5 receptors, and this property delays washout from the brain. For this reason, we scanned subjects for a relatively long time $(5 \mathrm{~h})$ but then had the problem of determining whether we had acquired too much data. We suspected radiometabolites were slowly accumulating in the brain for 2 reasons. First, the concentrations of radioactivity in the brain at late time points were always higher than those calculated, assuming that only parent radioligand entered the brain (Fig. 2). Second, as expected from the first reason, the value of $V_{\mathrm{T}}$ continued to increase with increasing scan durations, indicating the presence of radiometabolites (Fig. 4A). These probable metabolites were not merely spillover of radioactivity from the skull. The gradual overestimation of apparent distribution volume occurred fairly equally for all regions, including those that were both close to and distant from the skull.

We are unaware of a standard approach to determine optimal scan length when a radiometabolite is suspected of accumulating slowly in the brain. In the absence of clear guidance, we selected the scan length on the basis of when identifiability achieved its best (i.e., smallest) values. Identifiability was large with scan lengths of less than $1 \mathrm{~h}$ but achieved small $(\sim 1 \%)$ and relatively stable values by $2 \mathrm{~h}$ (Fig. 4A). Nevertheless, even if the 5-h values were correct, $V_{\mathrm{T}}$ calculated from $2 \mathrm{~h}$ would underestimate the true value by approximately only $11 \%$ (Fig. 4A).

\section{Defluorination}

On the basis of uptake of radioactivity in the skull, ${ }^{18} \mathrm{~F}-\mathrm{SP} 203$ is defluorinated in humans. Although difficult to estimate, this uptake is likely not problematic for quantitation of radioactivity in the brain. The extent of contamination depends in part on the relative concentration of radioactivity in the brain and skull. Furthermore, radioactivity attributed to uptake in the skull is composed of both spillover from the 
brain and radioactivity in the bone itself. The spillover from the brain declines as radioactivity washes out of the brain, but some additional source of radioactivity (presumably the ${ }^{18} \mathrm{~F}$-fluoride ion) accumulates during that time, so that the measured activity actually increases continually during the scan. Because we recommend scanning for no more than $120 \mathrm{~min}$, we estimated the cross-contamination in the skull and adjacent neocortex at this time of greatest potential contamination of the brain by radioactivity in the skull. At $120 \mathrm{~min}$, the measured concentration of radioactivity in the temporal cortex was $380 \%$ SUV and that in the skull was $150 \%$ (Fig. 2 ). To estimate the amount of cross-contamination, we assumed that all radioactivity measured in the skull in the first few minutes actually derived from the brain. At about $5 \mathrm{~min}$, radioactivity in the skull was $100 \%$ SUV and that in the temporal cortex was $600 \%$ SUV. The percentage spillover $(100 / 600=17 \%)$ would be the same in both directions (i.e., from brain to skull and vice versa). Thus, at $120 \mathrm{~min}$, assuming the same $17 \%$ spillover both ways with measured $380 \%$ SUV and $150 \%$ SUV activity in the temporal cortex and skull, the true activity would be $365 \%$ SUV and $88 \%$ SUV, respectively. In other words, only $15 \%$ SUV of the $380 \%$ SUV, or approximately $4 \%$ of the total measured cortical activity, was contamination from the skull. By this calculation, radioactivity in bone is unlikely a problem when one is estimating brain uptake of ${ }^{18} \mathrm{~F}-\mathrm{SP} 203$ with a scan duration of $120 \mathrm{~min}$.

\section{Comparison with Other Radioligands for mGluR5}

How does ${ }^{18} \mathrm{~F}$-SP203 compare with the previously reported compounds 3-fluoro-5-[(pyridine-3-yl)ethynyl]benzonitrile ( ${ }^{18}$ F-PEB), 3-fluoro-5-[(2-methyl-1,3-thiazol-4-yl)ethynyl]benzonitrile ( $\left.{ }^{18} \mathrm{~F}-\mathrm{MTEB}\right)$, and 3-(6-methyl-pyridin-2-ylethynyl)-cyclohex-2-enone- $O-{ }^{11} \mathrm{C}$-methyloxime $\left({ }^{11} \mathrm{C}-\mathrm{ABP} 688\right)$ ? Because we studied ${ }^{18} \mathrm{~F}-\mathrm{SP} 203$ in monkeys and humans, we can compare our radioligand with ${ }^{18} \mathrm{~F}-\mathrm{PEB}$ (4), which has been studied in monkeys (but not in humans), and with ${ }^{11} \mathrm{C}-\mathrm{ABP} 688$ (5), which has been studied in humans (but not in monkeys). In fact, all these compounds have similar diaryl alkyne structures and label the same receptor (i.e., mGluR5) with high specificity. The selectivity of these compounds for mGluR5 has been demonstrated with broad receptor screening assays (7) and in mice with knockout of the mGluR5 receptor (16). The relative utility of these radioligands as in vivo imaging agents depends strongly on their metabolism and on the kinetics of brain uptake and washout. These in vivo factors vary among species. For example, ${ }^{18} \mathrm{~F}-\mathrm{SP} 203$ has significant defluorination in monkeys so that we cannot easily compare its brain kinetics with that of the Merck compounds ${ }^{18} \mathrm{~F}-\mathrm{PEB}$ and ${ }^{18} \mathrm{~F}-\mathrm{MTEB}$. That is, the brain time-activity curves of ${ }^{18} \mathrm{~F}-\mathrm{SP} 203$ are strongly contaminated by high and accumulating radioactivity, presumably ${ }^{18} \mathrm{~F}$ fluoride ion, in adjacent bone (Fig. 4 in Siméon et al. (7)). With this caveat in mind, the peak uptake in the monkey brain of both ${ }^{18} \mathrm{~F}-\mathrm{PEB}$ and ${ }^{18} \mathrm{~F}-\mathrm{SP} 203$ is similar ( $\sim 500 \%$ SUV), and they have a similar washout rate. Nevertheless, ${ }^{18} \mathrm{~F}-\mathrm{PEB}$ is clearly superior to ${ }^{18} \mathrm{~F}$ SP203 for studies in monkeys because of the substantial defluorination of ${ }^{18} \mathrm{~F}-\mathrm{SP} 203$. With regard to human use, both 
${ }^{18} \mathrm{~F}-\mathrm{SP} 203$ and ${ }^{11} \mathrm{C}-\mathrm{ABP} 688$ have relative advantages and disadvantages. First, the shorter half-life of ${ }^{11} \mathrm{C}(20 \mathrm{~min})$, compared with ${ }^{18} \mathrm{~F}(110 \mathrm{~min})$, allows 2 studies to be performed in a subject in $1 \mathrm{~d}$ using ${ }^{11} \mathrm{C}-\mathrm{ABP} 688$. Such repeated scans are useful to study the acute effects of drug administration and are better tolerated by the subject, because the arterial line can remain in place and need not be inserted twice. Nevertheless, the longer half-life of ${ }^{18} \mathrm{~F}$ more easily allows the radioligand to be distributed to distant PET centers. Second, the peak uptake of ${ }^{18} \mathrm{~F}-\mathrm{SP} 203(\sim 580 \%$ SUV) is 4-fold higher than that of ${ }^{11} \mathrm{C}-\mathrm{ABP} 688(\sim 150 \%-200 \%$ SUV) and is a clear advantage of the ${ }^{18} \mathrm{~F}$-labeled radioligand. Third, radiometabolite(s) of ${ }^{18} \mathrm{~F}-\mathrm{SP} 203$ likely accumulating in the brain are a clear disadvantage of this radioligand. Nevertheless, because the values of $V_{\mathrm{T}}$ from $2 \mathrm{~h}$ of data are only $11 \%$ smaller than those from $5 \mathrm{~h}$ of data, the contribution of radiometabolites is suggested to be relatively small. In fact, if we had acquired images for only $2 \mathrm{~h}$, which is commonly done, we would not even have become aware of this problem. In addition, we do not know whether ${ }^{11} \mathrm{C}$-ABP688 has a similar problem with radiometabolites, because time-stability data of $V_{\mathrm{T}}$ have not been reported for ${ }^{11} \mathrm{C}-\mathrm{ABP} 688$.

\section{CONCLUSION}

This initial evaluation of ${ }^{18} \mathrm{~F}-\mathrm{SP} 203$ in healthy human subjects showed that defluorination is relatively low and that brain uptake can be robustly calculated as distribution volume, which is an index of receptor density. ${ }^{18} \mathrm{~F}-\mathrm{SP} 203$ should be useful for within-subject studies, such as receptor occupancy of therapeutic agents that, like SP203, are negative allosteric modulators. The values of distribution volume for ${ }^{18} \mathrm{~F}-\mathrm{SP} 203$ were well identified and had relatively small variation in this group of 7 healthy subjects. If these results are confirmed in larger samples, ${ }^{18} \mathrm{~F}-\mathrm{SP} 203$ may also have good sensitivity for between-subject studies, such as differences between patients and healthy subjects.

\section{ACKNOWLEDGMENTS}

We thank Maria Desiree Ferraris Araneta, Amanda Farris, Ed Tuan, Pavitra Kannan, and the staff of the PET department for successful completion of the studies, and we thank PMOD Technologies (Adliswil, Switzerland) for providing its image analysis and modeling software. This research was supported by the Intramural Program of NIMH (project Z01MH-002795-04).

\section{REFERENCES}

1. Conn PJ, Pin JP. Pharmacology and functions of metabotropic glutamate receptors. Annu Rev Pharmacol Toxicol. 1997;37:205-237.

2. Slassi A, Isaac M, Edwards L, et al. Recent advances in non-competitive mGlu5 receptor antagonists and their potential therapeutic applications. Curr Top Med Chem. 2005;5:897-911.

3. Pellicciari R, Costantino G, Marinozzi M, Macchiarulo A, Camaioni E, Natalini B. Metabotropic glutamate receptors: structure and new subtype-selective ligands. Farmaco. 2001;56:91-94.

4. Hamill TG, Krause S, Ryan C, et al. Synthesis, characterization, and first successful monkey imaging studies of metabotropic glutamate receptor subtype 5 (mGluR5) PET radiotracers. Synapse. 2005;56:205-216.

5. Ametamey SM, Treyer V, Streffer J, et al. Human PET studies of metabotropic glutamate receptor subtype 5 with ${ }^{11} \mathrm{C}$-ABP688. J Nucl Med. 2007;48:247-252.

6. Treyer V, Streffer J, Ametamey SM, et al. Radiation dosimetry and biodistribution of ${ }^{11} \mathrm{C}$-ABP688 measured in healthy volunteers. Eur J Nucl Med Mol Imaging. 2008;35:766-770.

7. Siméon FG, Brown AK, Zoghbi SS, Patterson VM, Innis RB, Pike VW. Synthesis and simple ${ }^{18} \mathrm{~F}$-labeling of 3-fluoro-5-(2-(2-(fluoromethyl)thiazol-4yl)ethynyl)benzonitrile as a high affinity radioligand for imaging monkey brain metabotropic glutamate subtype-5 receptors with positron emission tomography. J Med Chem. 2007;50:3256-3266.

8. Gandelman MS, Baldwin RM, Zoghbi SS, Zea-Ponce Y, Innis RB. Evaluation of ultrafiltration for the free-fraction determination of single photon emission computed tomography (SPECT) radiotracers: beta-CIT, IBF, and iomazenil. J Pharm Sci. 1994;83:1014-1019.

9. Tzourio-Mazoyer N, Landeau B, Papathanassiou D, et al. Automated anatomical labeling of activations in SPM using a macroscopic anatomical parcellation of the MNI MRI single-subject brain. Neuroimage. 2002;15:273-289.

10. Innis RB, Cunningham VJ, Delforge J, et al. Consensus nomenclature for in vivo imaging of reversibly binding radioligands. J Cereb Blood Flow Metab. 2007; 27:1533-1539.

11. Burger C, Mikolajczyk K, Grodzki M, Rudnicki P, Szabatin M, Buck A. JAVA tools quantitative post-processing of brain PET data [abstract]. J Nucl Med. 1998;39(suppl):277P.

12. Nishikawa H, Hashino A, Kume T, Katsuki H, Kaneko S, Akaike A. Involvement of direct inhibition of NMDA receptors in the effects of sigma-receptor ligands on glutamate neurotoxicity in vitro. Eur J Pharmacol. 2000;404:41-48.

13. Hawkins RA, Phelps ME, Huang S-C. Effects of temporal sampling, glucose metabolic rates, and disruptions of the blood-brain barrier on the FDG model with and without a vascular compartment: studies in human brain tumors with PET. J Cereb Blood Flow Metab. 1986;6:170-183.

14. Carson RE. Parameter estimation in positron emission tomography. In: Phelps ME, Mazziotta JC, Schelbert HR, eds. Positron Emission Tomography and Autoradiography: Principles and Applications for the Brain and Heart. New York, NY: Raven Press; 1986:347-390.

15. Patel S, Hamill TG, Connolly B, Jagoda E, Li W, Gibson RE. Species differences in mGluR5 binding sites in mammalian central nervous system determined using in vitro binding with [ ${ }^{18}$ F]F-PEB. Nucl Med Biol. 2007;34:1009-1017.

16. Ametamey SM, Kessler LJ, Honer M, et al. Radiosynthesis and preclinical evaluation of ${ }^{11} \mathrm{C}-\mathrm{ABP} 688$ as a probe for imaging the metabotropic glutamate receptor subtype 5. J Nucl Med. 2006;47:698-705. 